\title{
Impact of mild-moderate mitral regurgitation on outcomes of isolated aortic valve replacement
}

\author{
Rong Hui (Misté) Chia ${ }^{1 *}$, Julian A Smith', Rory SJ Wolfe², Aubrey A Almeida' \\ From World Society of Cardiothoracic Surgeons 25th Anniversary Congress, Edinburgh \\ Edinburgh, UK. 19-22 September 2015
}

\section{Background/Introduction}

The impact of mitral regurgitation (MR) severity on patients undergoing aortic valve replacement (AVR) for aortic stenosis remains unclear.

\section{Aims/Objectives}

This study evaluated the effects of mild or moderate MR on outcomes of isolated AVR for aortic stenosis.

\section{Method}

Clinical outcomes evaluated were postoperative complications; length of stay in intensive care unit (ICU); 30-day and late mortality; and the degree of $M R$ improvement on echocardiograms after AVR. MR severity was defined according to the European Association of Echocardiography recommendations. Medium-term functional outcome was assessed using the Short Form36 quality of life (QoL) questionnaire.

\section{Results}

Eighty-nine patients received isolated AVR for significant aortic stenosis from August 2008 to September 2014, of which 53 patients had co-existing mild MR while 36 had moderate MR. Both groups were similar prior to surgery, except in the incidence of concomitant aortic regurgitation (28\% versus $17 \%$ ). Median follow-up time for postoperative echocardiograms and QoL assessment were 1 and 3 years respectively.

The odds of postoperative complications were greater in the moderate MR patients although this may have been a chance observation (OR, 2.3; $\mathrm{p}=0.3)$. Mild $M R$ patients had fewer postoperative complications (mean of $2 \pm 2$ versus $3 \pm 3$ complications; $p=0.04$ ). There was no significant difference in the odds of mean duration of ICU-stay (mean $3 \pm 3$ versus $4 \pm 4$ days; $\mathrm{p}=0.4$ ) or 30-day mortality between groups (OR, 1.6; $\mathrm{p}=0.5)$. Difference in late-mortality was insignificant (HR, 1.2; $\mathrm{P}=$ $0.5)$. Based on available postoperative echocardiograms, mild MR $(\mathrm{n}=37)$ worsened in MR grade by $0.1 \pm 0.5$ whereas moderate MR $(n=27)$ improved by $0.4 \pm 0.6$ in MR grade after AVR $(\mathrm{p}=0.01)$. Fewer patients with mild MR made an improvement in MR grade postoperatively ( $27 \%$ versus $59 \%$ ). Only $79 \%$ and $72 \%$ of the patients in mild and moderate MR groups respectively were alive at the time of survey. At follow-up, both groups had similar QoL.

\section{Discussion/Conclusion}

Clinical and medium-term functional outcomes were similar in patients with mild or moderate MR undergoing isolated AVR.

\section{Authors' details \\ ${ }^{1}$ Department of Cardiothoracic Surgery, Monash Health, Melbourne, Victoria, 3168, Australia. ${ }^{2}$ Department of Epidemiology and Preventive Medicine,} Monash University, Melbourne, Victoria, 3004, Australia.

Published: 16 December 2015

doi:10.1186/1749-8090-10-S1-A180

Cite this article as: Chia et al:: Impact of mild-moderate mitral

regurgitation on outcomes of isolated aortic valve replacement. Journal of Cardiothoracic Surgery 2015 10(Suppl 1):A180. 